\title{
3D-Based Resources Fostering the Analysis, Use, and Exploitation of Available Body Anthropometric Data
}

\author{
Alfredo BALLESTER*, Eduardo PARRILLA, Jordi URIEL, Ana PIEROLA, Sandra ALEMANY, \\ Beatriz NACHER, Jorge GONZALEZ, Juan Carlos GONZALEZ \\ Instituto de Biomecánica de Valencia (IBV), Spain \\ http://dx.doi.org/10.15221/14.237
}

\begin{abstract}
Today, there is an increasing availability of human body 3D data and an increasing number of anthropometric owners. This is due to the fact of the progressive conduction of large national surveys using high resolution 3D scanners and due to the increasing number of low-cost technologies for acquiring body shape with electronic consumer devices like webcams, smartphones or Kinect. However, the commercial use and exploitation in industry of digital anthropometric data is still limited to the use of 1D measurements extracted from this vast 3D information. There is a lack of universal resources enabling: to conjointly use and analyse datasets regardless from the source or type of scanning technology used, the flexible measurement extraction beyond pre-defined sets, and the analysis of the information contained in human shapes. This paper presents four software tool solutions aimed at addressing different user profiles and needs regarding the use and exploitation of the increasing number of 3D anthropometric data
\end{abstract}

Keywords: Sizing Surveys, Databases, Measurements, Body Scanning, 3D Surface, Anthropometry, Shape Registry, Homologous Representation, Topology Harmonization, Shape Analysis, Avatar, Human Body Shape

\section{Introduction}

Over the last decades, individual 3D and measurement anthropometric information has been generated around the world. Since 1999, many large-scale 3D full body scanning surveys have been conducted using high precision scanning technologies. During last years, due to the commercialization of scanning technologies, several product customization initiatives have been setting up measurement devices at points of sale, facilitating the order and manufacture of made to measure garment and orthopaedic products, among others. Moreover, the recent appearance of low cost scanning and 3D printing technologies is boosting the acquisition of 3D human data of full bodies both by individual users and online retailers.

Despite the increasing availability of 3D data and the increasing number of 3D data owners, its use and exploitation is still limited to pre-defined sets of measurements that are extracted using the applications provided by scanning technology supplier. There is a lack of universal resources enabling:

(i) to conjointly use and analyse datasets regardless from the source or type of scanning technology used,

(ii) the flexible measurement extraction beyond closed sets of measurements, and

(iii) the analysis of the vast information contained in shapes, which is currently gathered in 3D scans but disregarded for commercial and industrial applications.

During the last three years, IBV has been working in the development of methods and software enabling to bridge the existing gap between existing 3D human shape data and its use in different ergonomic and non-ergonomic applications. IBV has developed a series of technologies, based on the principles of the homology of human body references and on the use of the principal components of body shape.

*corresponding author alfredo.ballester@ibv.upv.es, www.ibv.org 
These technologies are aimed at fostering the use and broadening the scope of current use of 3D anthropometric data. These technologies can be divided into three groups:

- Body scan data harmonization, that enables the creation of homologous and manageable representations of each individual scan including skeletons from most of the scanning technologies;

- 1D measurement data enhancement to 3D, that enables the recreation 3D human shapes from a set of 1D measurements; and

- 3D shape analysis, that enables to relate the principal components of shape to a set of measurements.

This paper presents four software tools aimed at addressing different user profiles and needs regarding the use and exploitation of the increasing number of 3D anthropometric data, including some application examples for each of them:

- Data harmonization package for 3D-database owners and scanning technology suppliers

- Data enhancement package for measurement-database owners

- Data-driven avatar creation package for virtual try-on applications

- Shape analysis tools for data revalue and product design

\section{Enabling technologies}

The following paragraphs briefly describe the scientific basis underlying the technologies that have been used to build the software packages and services presented in this paper.

\subsection{Harmonization of body scans data}

The harmonization of 3D scan data consists of fitting the same template mesh topology equipped with a skeleton to the surface of each of the raw scans of a database, obtaining a homologous structured representation of all the individuals in the database with point-to-point correspondence (Figure 1). Then, the skeleton can be used to repose all the individuals of the database to the same posture.

The template structure used by IBV was developed under the EUROFIT project $[1,2,3]$. It is constituted by a single closed mesh surface made up of 49.530 vertices, 99.056 triangle faces and a 17-bone and 14-joint skeleton as depicted in Figure 2 [4]. Its structure features a high local density providing sufficient resolution for accurate representation of body shapes from 3D raw scan data (i.e. point-to-point average error below $0.5 \mathrm{~mm}$ ) and light enough to follow multivariate analysis of large target population selections (i.e. thousands of individuals). The process consists of three main steps: preprocessing, template-fitting, and posture harmonization.

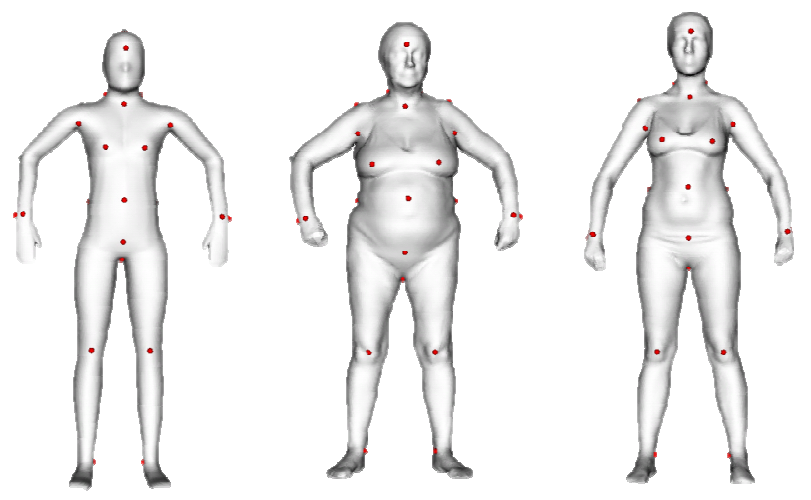

Figure 1: Homology concept: point-to-point correspondence

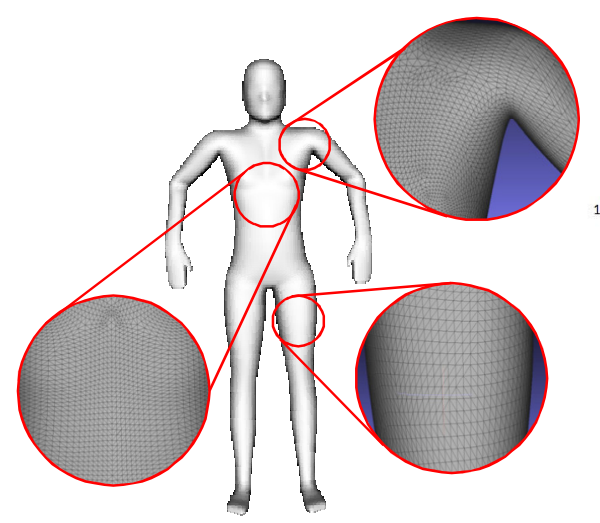

Figure 2: Template reference structure and skeleton 
- Pre-processing is aimed at obtaining a single closed surface from the raw scan which contains noise, missing information and redundancies (Figure 4) by using Poisson's reconstruction algorithms [5], surface fairing [6] and identifying missing information areas in the body which will receive an special treatment in the template-fitting step. Examples of pre-processed scans are provided in Figure 3.

- Template-fitting [7] consists of iteratively deforming the template mesh until it matches the pre-processed surface using a set of anatomical landmarks as the main reference in the fitting process (Figure 5). The methods developed by IBV are adapted implementations of the methods presented by Allen et al. [8], Amberg et al. [9], Summers and Popovich [10] and Liepa [6]. One of the key advantages of this implementation is the automated reconstruction of slit areas where actual information of the scan is missing like the crotch and the armpits (Figure 6).

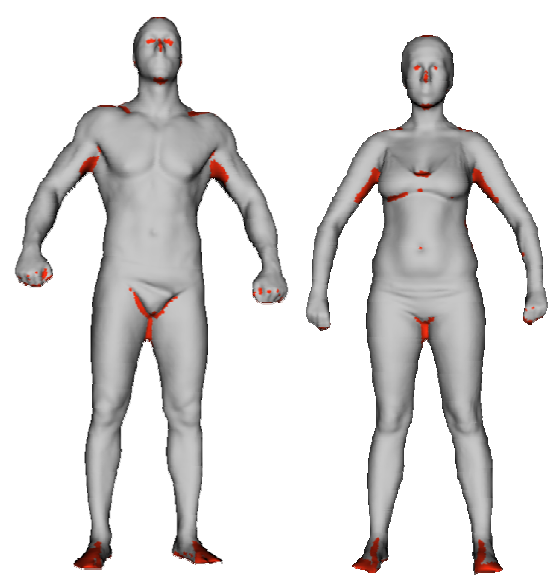

Figure 3: images of pre-processed scans
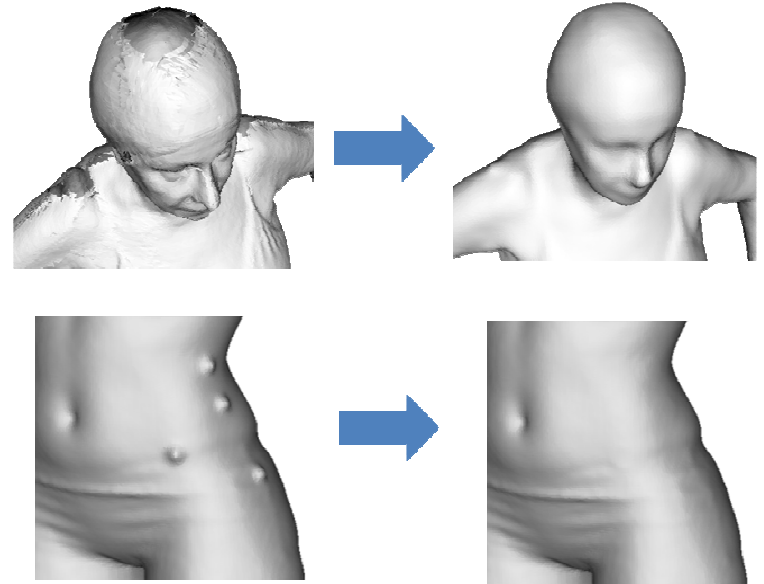

Figure 4: hole-filling and landmark removal
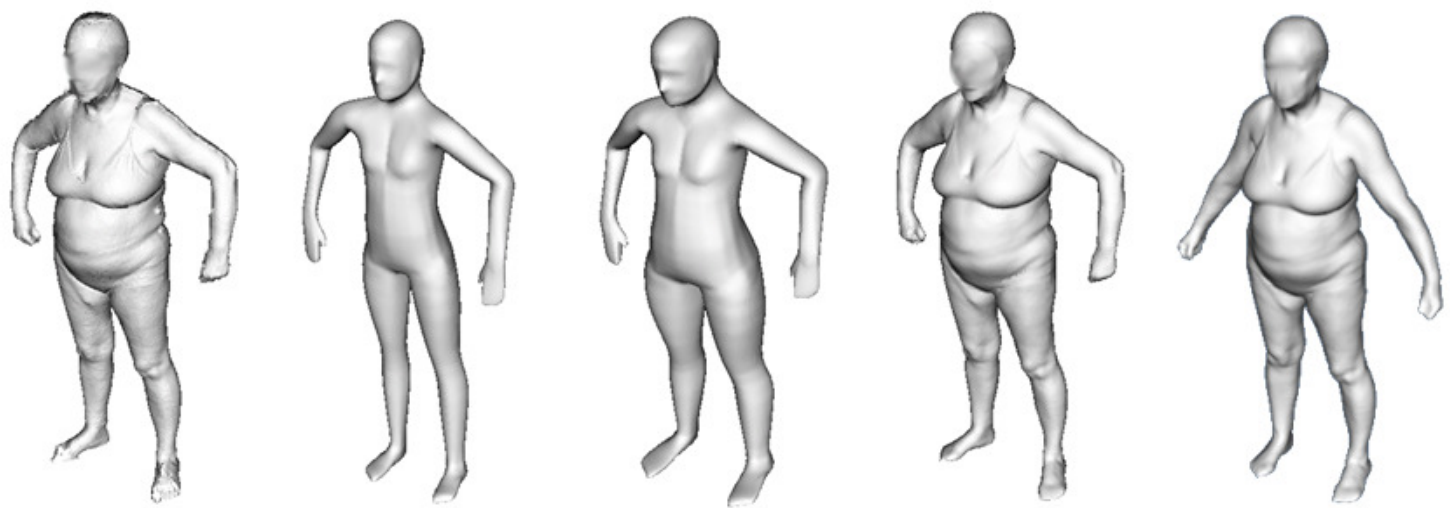

Figure 5: From left to right: Raw Scan; Template; Posed template; Homologous Model and Posture-corrected Model
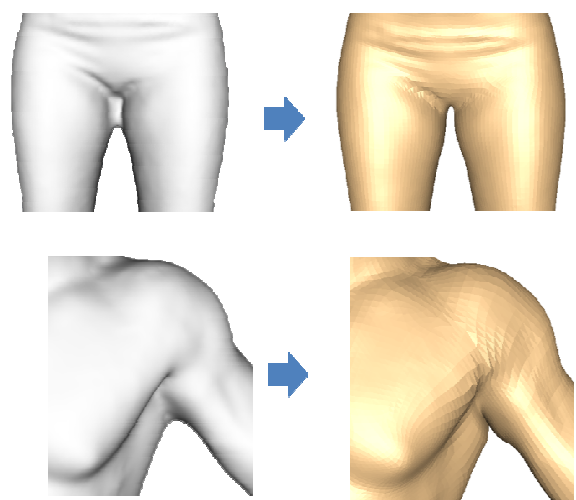

Figure 6: Automated natural slit modelling
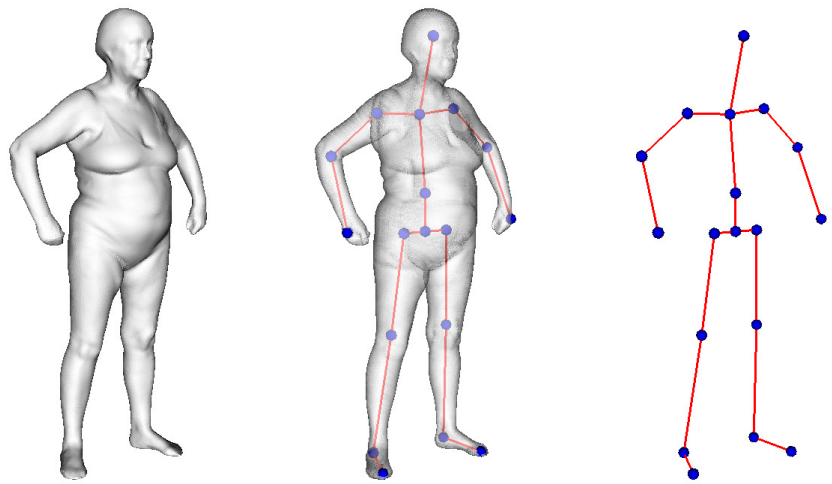

Figure 7: Harmonized homologous mesh with skeleton 
- Posture harmonization consists of equipping each of the homologous representations with the anatomical skeleton of the template and then defining a common posture restriction for the bones/joints of the skeletons of all individuals [11]. The skeleton is transferred from the template through homology (point-to-point correspondence) using the mean value coordinates method [12] adapted to the 3D space. After the transfer, skin of the Homodel is attached to the skeleton (Figure 7). The weights of each bone on the Homodel skin (vertices) are recalculated. In this way, the skin of the Homodel is deformed according to the skeleton posture [13]. Skin and skeleton are then moved to adopt the neutral posture defined following an adapted implementation of Mazany and Svoboda [14]. Images of the results of posture harmonization are provided in Figure 8.

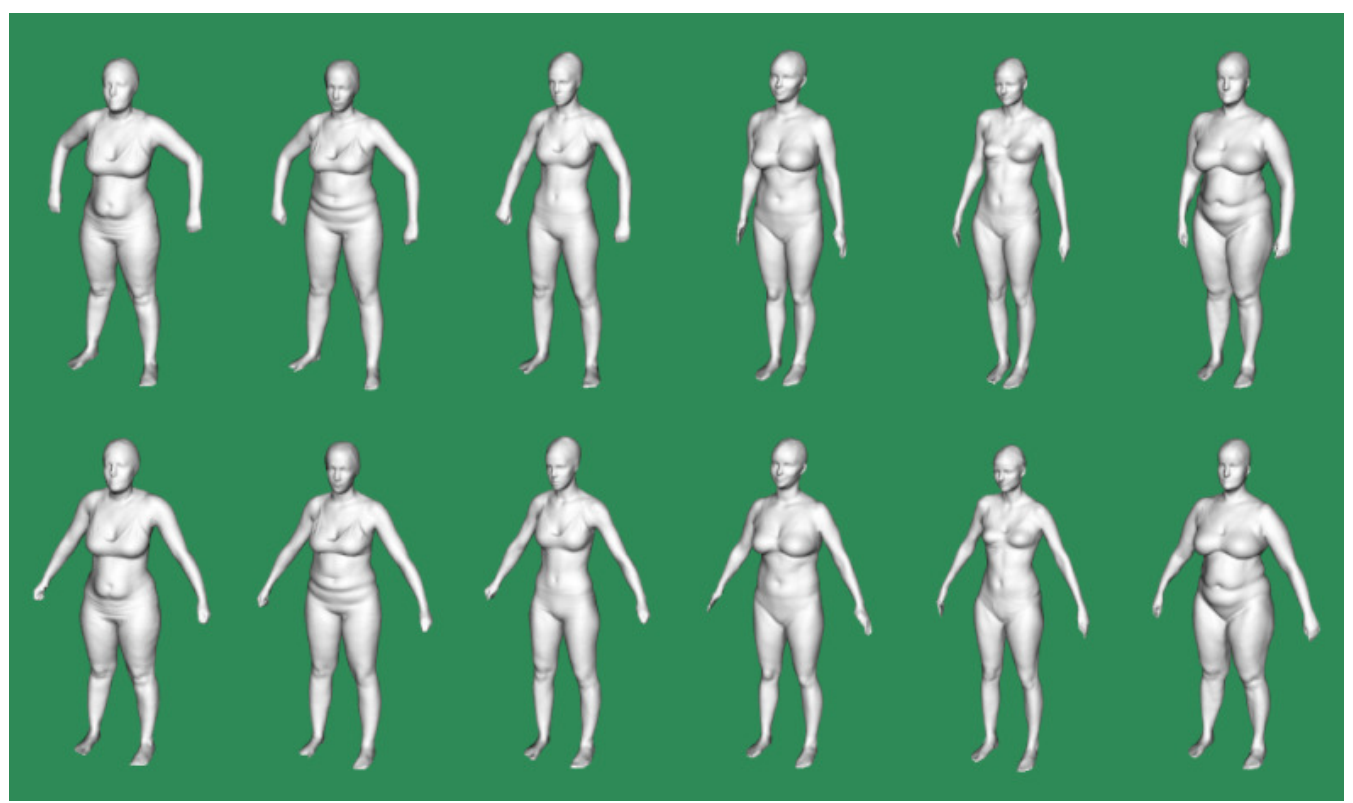

Figure 8: Top line: Homologous models from Spanish and German Surveys in survey postures. Bottom line: same homologous models after posture harmonization.

Algorithms and software developed for 3D data harmonization has been tested for both laser-scan technology samples as well as for structured-light technology from CAESAR $[15,16]$, Spanish $[17,18]$ and Portuguese [20] National 3D Surveys. Some examples are provided in Figure 9.

Since this is a procedure that has to be massively applied to large individual databases (i.e. from 2.000 to 20.000 individuals), it has been developed to be fully automatic, just requiring an initial pre-configuration that consists of selecting a set of anatomical references/landmarks to be used (which number can range from 15 to 70 , typically around 30).

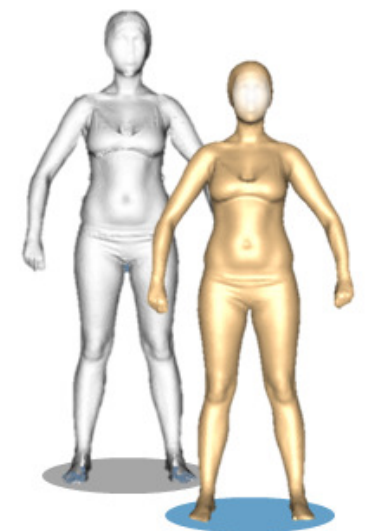

Size Spain

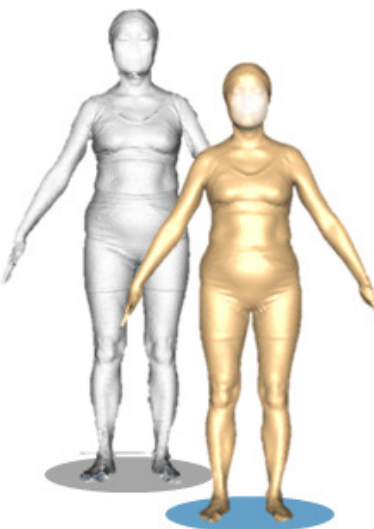

CAESAR US

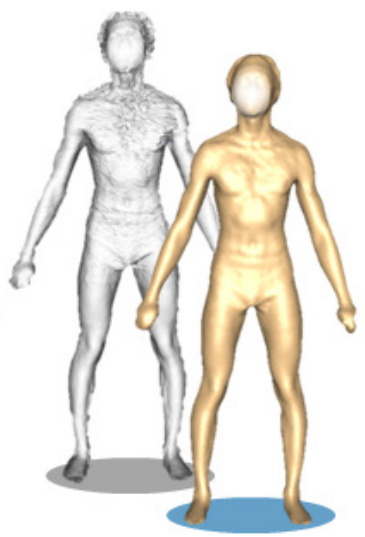

Size Portugal

Figure 9: Examples of homologous body representations from different scan technologies and postures 


\subsection{Shape Analysis using Principal Component Analysis}

In order to analyze and use shape information from large samples of 3D homologous models describing human body shape, complex analyses must be applied. Mesh deformation and principal component analysis (PCA) are the most common methods used in 3D body shape analysis [21]. PCA is the most suitable since it provides the statistical control required for applying results to engineering and design problems. PCA is a statistics-based method that enables to smartly synthesize huge volumes of information $[22,23,24]$. Moreover, the fact of using homologous and posture-harmonized models facilitates the interpretation of the meaning of each component. Figure 10 shows a comparison of the first three Principal Components of shape for a female sample before and after the posture harmonization.

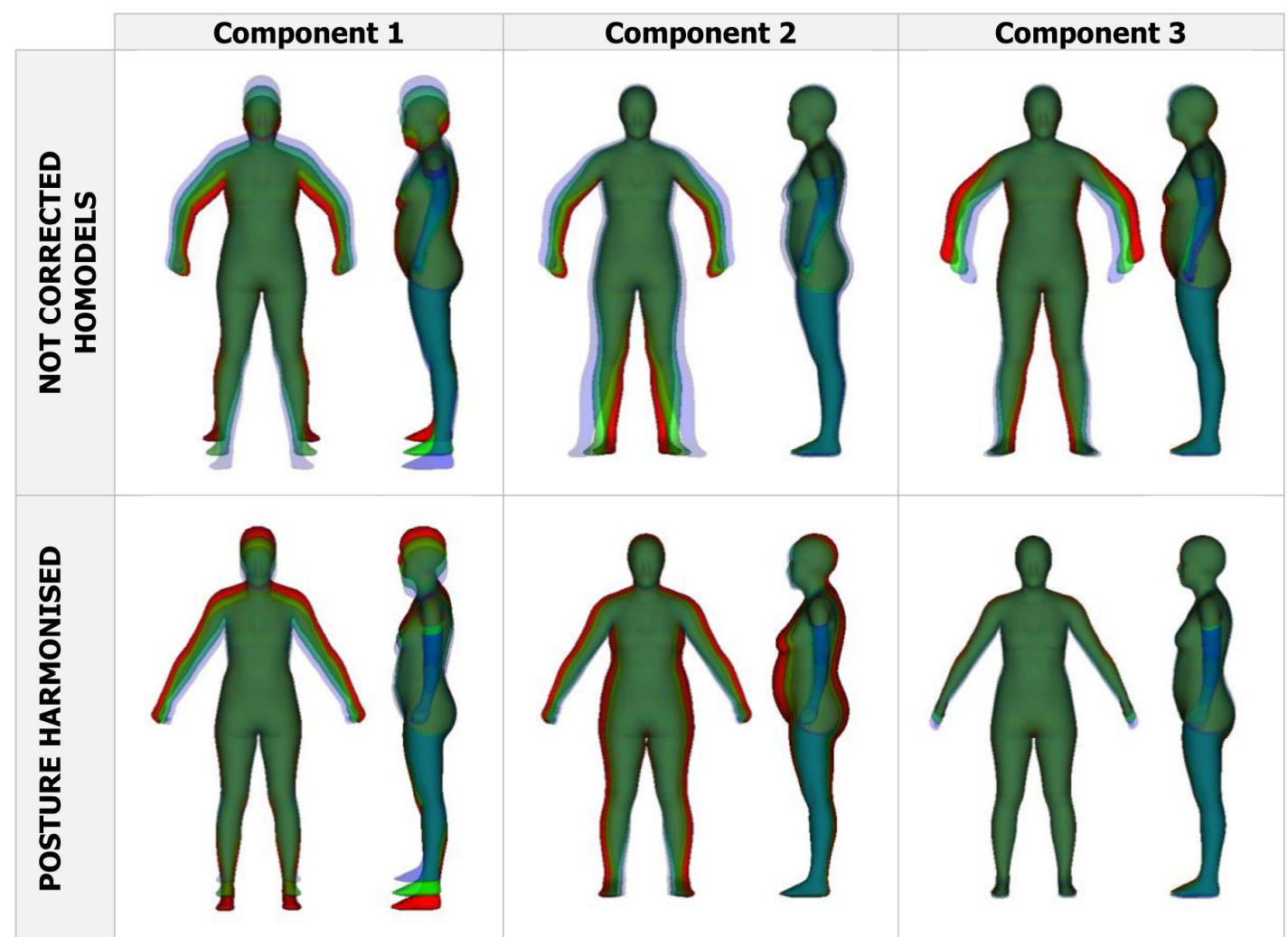

Figure 10: Top line: PCA components 1, 2 and 3 with non-manipulated Homodels (9.000 Spanish women sample). Bottom line: PCA modes 1, 2 and 3 with posture manipulated Homodels (9.000 Spanish sample).

\subsection{Enhancement of 1D measurement data to 3D homologous models}

Once a database of human homologous shapes has been created, and it has been synthesized to its Principal Components, it is possible to create a model that relates a set of body measurements with the combination of the PCA scores that match them [25]. Additionally, this shape can be optimized by iterative measurement and search of the target measurements. Optimization can be achieved by solving a minimization problem using gradient-descent methods [26] where the variables are the PCA scores minimizing the deviations from the desired measurements. The performance of the former approach in our implementation achieves maximum differences between the target value and actual value of $1-5 \mathrm{~cm}$ depending on the combination of measurements, while the optimized implementation can achieve maximum error values in the order of $0.01 \mathrm{~cm}[1,27]$.

\section{Software tools and application examples}

The following paragraphs describe the features and some examples of the tools developed. 


\subsection{Data harmonization package for 3D database owners}

As previously mentioned, raw scan data is difficult to exploit, treat and analyze. Companies and organizations which own large national/regional databases often lack the means for processing and analyzing raw scan datasets beyond the tools originally provided by the scan technology suppliers, which are normally limited to a measurement extraction.

This tool package consists of three standalone software applications for massive operations on large 3D scan samples in standing posture:

a. automated creation of watertight 3D homologous representations of each scan including posture correction of the dataset and skeleton rigging for each individual scan;

b. pre-defined measurement extraction for 100 measurements based on ISO 7250 [28] and 8559 [29]; and

c. new measurement definition for product specific measurements.

These tools can be adapted and pre-configured for the specific needs of the database owner, according to the type of scanner used and the number of anatomical references to be used. This technology has already been used for the creation of the Spanish female, male and children 3D homologous databases by IBV.

\subsection{D data enhancement package for measurement database owners}

Most of the anthropometric databases available consist of collections of individual measurement sets. This tool package consists of three standalone software applications for:

a. recreation of individual 3D representations with skeleton from individual sets of 1D measurements, which is flexible and can be configured to work with different input sets;

b. pre-defined measurement extraction based on ISO 7250 and 8559; and

c. new measurement definition to extend pre-existing measurements.

The main advantage of this tool is that measurement database owners will be able to re-create the 3D body shape of each individual of their database and interrogate them to extract an estimation of any additional measurement not included in the original set.

These tools can be adapted and configured according to the set of input measurements that the database owner defines, ranging from 5 to more than 100 . The number of extended measurements to be extracted can also be configured upon request. Current shape reconstruction base is made up of more than 11.000 individual scans, which guarantees a realistic estimation of real human body shapes.

\subsection{Data-driven avatar creation package for virtual try-on applications}

Most of the garment virtual try-on solutions in the market are based on the recreation of a videogame-like parametric avatar that fulfils a number of measurements and parameters provided by the user such as height, weight and several perimeters [30,31,32]. One of the main problems of these solutions is that user acceptance is reduced due to the low realism of the body shapes generated.

This software solution consists of a library addressed to IT developers or online retailers that offer virtual try-on solutions and online size recommendation systems enabling to recreate realistic data-driven 3D human representations with skeleton from a set of measurements as well as to extract 1D measurements.

In order to illustrate the potential of the data-driven recreation of human shapes from a small set of 1D measurement data, Figure 11 compares a 3D representation created with our solution, with MakeHuman [33] and with Optitex Online Avatar [34], all based on 11 measurements taken from two real women (Figure 12). 


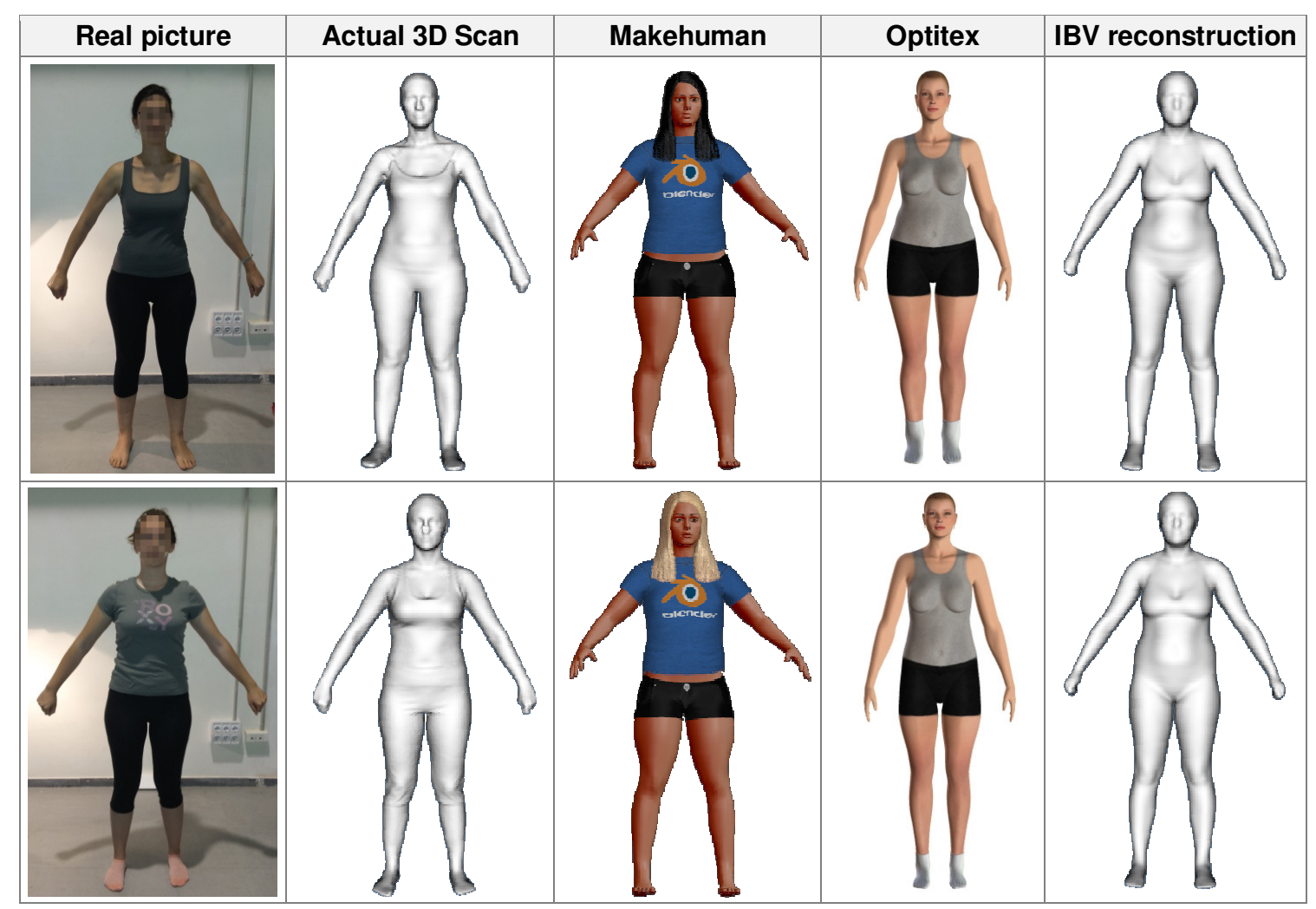

Figure 11: Comparison of different avatar creation tools from measurements based on real female examples

\begin{tabular}{|l|r|r|}
\hline \multicolumn{1}{|c|}{ Measurement } & Subject A & Subject B \\
\hline Height & 1670 & 1690 \\
\hline Knee height & 445 & 468 \\
\hline Bust girth & 920 & 942 \\
\hline Underbust girth & 770 & 812 \\
\hline Waist girth & 900 & 877 \\
\hline Hip girth & 1102 & 1037 \\
\hline Thigh girth & 625 & 614 \\
\hline Calf girth & 381 & 365 \\
\hline Ankle girth & 298 & 244 \\
\hline Upper arm girth & 287 & 297 \\
\hline Wrist girth & 155 & 154 \\
\hline
\end{tabular}

Figure 12: Table of measurements extracted from two women and used as the input for the creation of their respective avatars

\subsection{Shape analysis tools for data revalue}

As previously stated, there is a lack of universal resources enabling us to analyse datasets regardless from the source or type of scanning technology used. These tools are aimed to overcome existing barriers to the exploitation of shape information and the use of special user-defined measurements. It is addressed to specialised entities in the research of human body metrics and shape or the providers of consultancy services based on this kind of data.

It consists of a set of standalone tools aimed at:

a. the calculation of Principal Components of human shape data,

b. the creation of the representative 3D shapes from the shape components and its visualization,

c. pre-defined measurement extraction based on ISO 7250 and 8559,

d. the definition of new measurement for product specific measurements, and

e. establishing and modelling measurement-component relations. 
An example of the potential of the application of this technology for product design and development was conducted within the EUROFIT project [1,4], were Principal Components of Spanish women lower body shape were used to create a new three-dimensional sizing system of the female work trousers developed by the company Iturri S.A. Principal components were used to identify and quantify the intrinsic shape variations related to the key design parameters of the trouser pattern. This new size span was built following the main shape variations identified as relevant which were:

- Body girths: coincident with component 2 of the shape space (Figure 13). Since it included the largest dimensional variation, it was used to create the main size span that ranged from size 44 to 54.

- Lower body length: coincident with component 1 of the shape space. Its range of variation was divided into 3 steps, leading to Short, Medium and Long.

- Hip-waist morphotypes: coincident with mode 4 of the shape space (Figure 14). Its range of variation was divided into 3 steps, leading to Shaped, Regular and Straight body types.
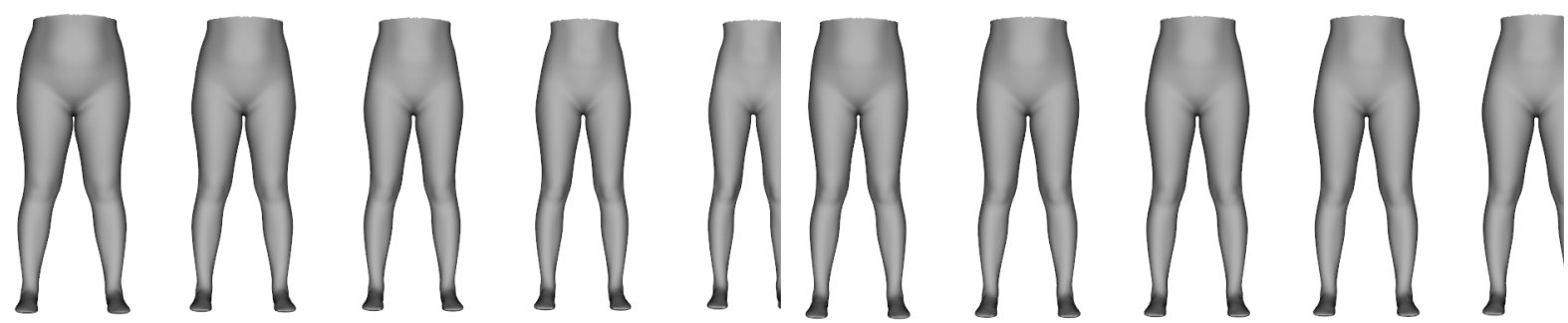

Figure 13: Second component of shape (girths)

Figure 14: first component of shape (morphotype)
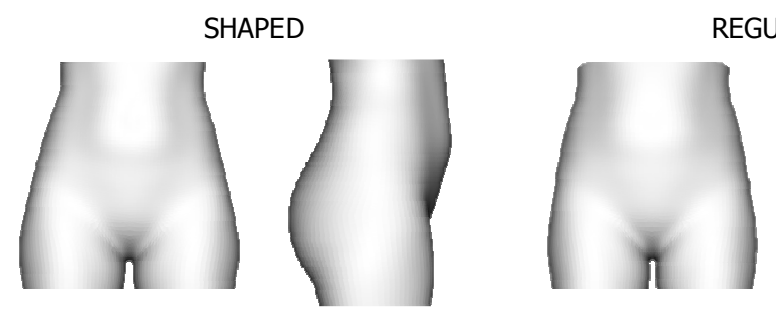

REGULAR

STRAIGHT

Figure 15: the three hip-waist morphotypes selected for Iturri sizes
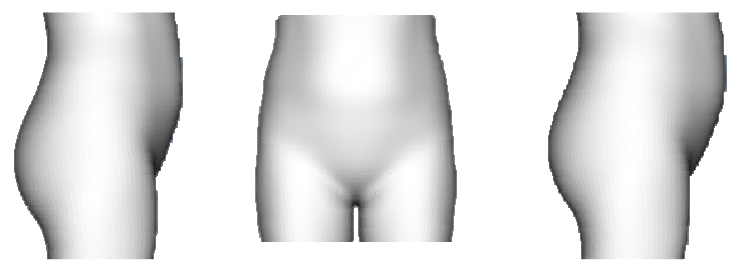

Within the project, product samples were manufactured and the new size span was assessed against the original one. Assessment was focused (i) on population coverage against the Spanish database of women in working age (aged 18-65) and (ii) on comfort and fitting provided.

Regarding population coverage, assessment was conducted using the EUROFIT portal prototype [] the results showed that the new size span had theoretical population coverage of 6 times higher than the original size span.

Regarding the comfort and fitting assessment, Iturri S.A manufactured a sample of trousers in several sizes (Figure 16) and a fitting test involving 50 users was conducted, were both expert and user evaluations of the trousers were made on a blind test. Results showed that the introduction of morphotypes variations improved both fitting and comfort of the users and facilitated size allocation of the trousers (Figure 17). 


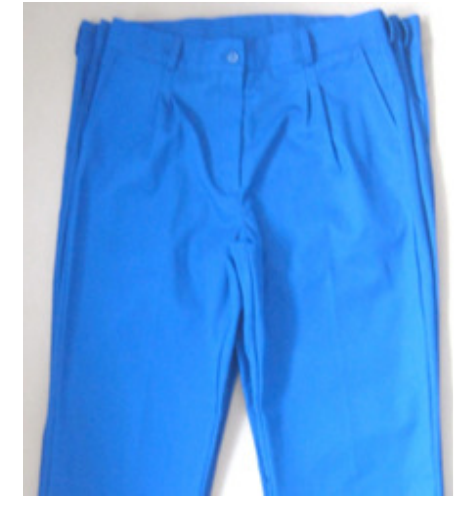

Figure 16: Work trouser sample in size 42 for the 3 morphotypes

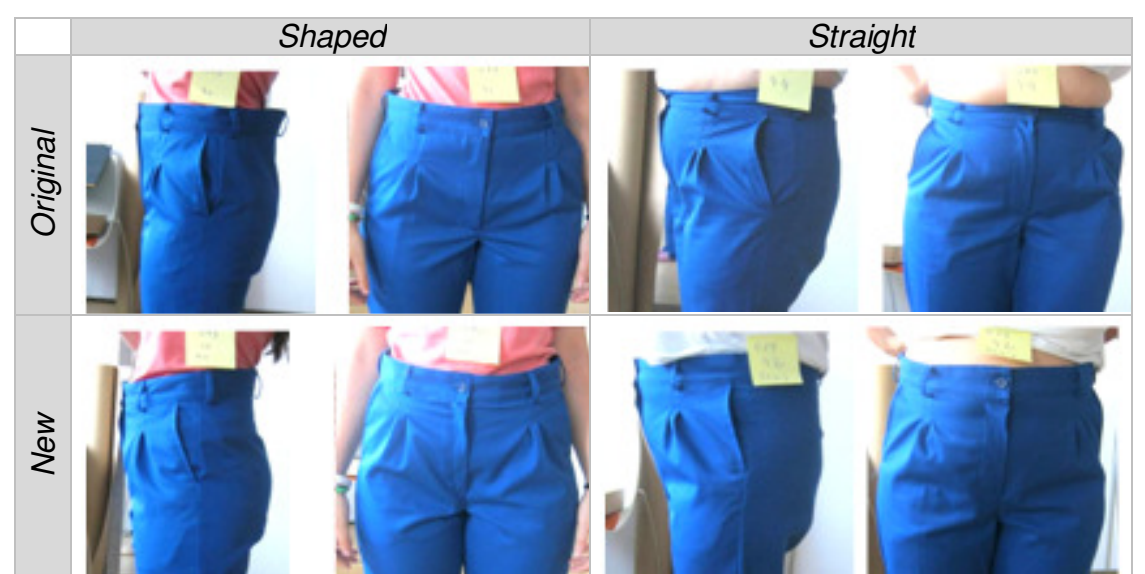

Figure 17: images of the user testing comparing original vs. new series

\section{Conclusions}

The results presented in this paper are relevant for the advancement in $3 \mathrm{D}$ data harmonization, treatment and analysis, in particular for the advancement in shape analysis. This paper introduces a fully automated process for the cleaning, completion and structuring of 3D human data. Moreover, it introduces automated tools for the posture harmonization enabling the cross analysis among 3D databases in standing posture presenting small differences in terms of arms and leg postures. The data-driven recreation offers, on the one hand, the possibility of creating more shape-realistic representations of individual people, and on the other hand, the possibility of obtaining estimated measurements of individuals not originally included in 1D databases.

Despite the method is at present optimized just for conducting small posture changes in standing posture, it sets the basis for the conduction of larger movements and has the potential to be extended to sitting posture. The fact that homologous models or data-driven avatars created with these methods can be equipped with a skeleton, also gives the possibility for using them in animation and motion.

These new tools will allow data owners, new scanning technology developers and product development companies in garment, orthotics and other sectors to improve the exploitation 1D and 3D data in the following ways:

- Database owners will be able to obtain more manageable databases, to conduct shape analyses, to create representative mannequins and to extract additional measurements beyond those pre-defined. Moreover it will be possible for them to use their data in cross-national studies.

- Virtual try-on tools developers and Specialised CAD developers will be able to create more realistic avatars and integrate them into their existing CAD, visualisation and try-on solutions.

- Scanning technology developers will be able to integrate harmonisation tools into the post-processing of their scanning software, being able to directly obtain watertight meshes of human shapes within the same scanning session.

- Product designers and manufacturers: will benefit from the use of shape analysis and representative avatar creation tools integrating them into their product development processes for improving comfort and fit. 


\section{Acknowledgements}

The authors would like to thank Iturri S.A. for their participation in EUROFIT project and for permitting the inclusion in this paper of the results of the study conducted for the development, manufacturing and testing of the new size span of corporate trousers. The authors also thank SIZING-SUDOE and EUROFIT project partners for making possible the successful completion of this project.

\section{References}

[1] Trieb, R.; Ballester, A.; Kartsounis, G.; Alemany, S.; Uriel, J.; Hansen, G.; Sanguinetti, M.; Genabith, M. EUROFIT: Integration, Homogenisation and Extension of the Scope of Large 2D Anthropometric Data Pools for Product Development.4th International Conference on 3D Body Scanning Technologies, Long Beach, CA, USA, November 2013.

[2] EUROFIT Project. FP7-ICT-2011-296116. "Integration, Homogenisation and Extension of the Scope of Anthropometric Data Stored in Large EU Pools", FP7, EC, http://www.eurofit-project.eu/

[3] Alemany, S.; Ballester A.,; Parrilla E.,; Uriel, J.; González, J.; Nácher, B.; González, J.C.; Page, A. Exploitation of 3D Body Databases for the Apparel Industry". 4th International Conference on 3D Body Scanning Technologies, Long Beach, CA, USA, November 2013.

[4] EUROFIT reference body template and skeleton. http://www.eurofit-project.eu/cms/upload/Public Downloads/Body reference package.zip

[5] Kazhdan, M., Bolitho, M., \& Hoppe, H. (2006, June). Poisson surface reconstruction. In Proceedings of the fourth Eurographics symposium on Geometry processing

[6] Liepa, P. (2003): "Filling holes in meshes". In Proceedings of the 2003 Eurographics/ACM SIGGRAPH symposium on Geometry processing (pp. 200-205). Eurographics Association.

[7] EUROFIT project - 3D scan registration. https://www.youtube.com/watch?v=JnmE5lgQvXI

[8] Allen, B., Curless, B., \& Popović, Z. (2003): "The space of human body shapes: reconstruction and parameterization from range scans". In ACM Transactions on Graphics (TOG) (Vol. 22, No. 3, pp. 587-594). ACM.

[9] Amberg, B., Romdhani, S., \& Vetter, T. (2007): "Optimal step nonrigid icp algorithms for surface registration". In Computer Vision and Pattern Recognition, 2007. CVPR'07. IEEE Conference.

[10] Sumner ,R. and Popovic, J., "Deformation Transfer for Triangle Meshes." SIGGRAPH 2004.

[11] EUROFIT project - Posture harmonisation. https://www.youtube.com/watch?v=wmDwjnEWGoQ

[12] Floater, M.S. 2003. Mean value coordinates. Comput. Aided Geom. Des. 20, 1, 19-27.

[13] Baran, I. and Popović, J., Automatic rigging and animation of 3D characters, ACM Transactions on Graphics (TOG), 26 (3), 72:1-72:8 July 2007.

[14] Mazany O., Svoboda T., Articulated 3D human model and its animation for testing and learning algorithms of multi-camera systems, Master Thesis, Czech Technical University, FEL, CTU-CMP-2007-02, 2007.

[15] Robinette, K.M., Daanen, H., Paquet, E.: The Caesar Project: A 3D Surface Anthropometry Survey. In: Proc. in 3D Digital Imaging and Modeling, pp. 380-386, 1999.

[16] Civilian American and European Surface Anthropometry Resource Project-CAESAR ${ }^{\text {B }}$. SAE International. http://store.sae.org/caesar/

[17] Alemany, S., Gonzàlez, J.C., Nàcher, B. Soriano, C., Arnàiz, C. and Heras, A., Anthropometric Survey of the Spanish Female Population Aimed at the Apparel Industry. International Conference on 3D Body Scanning Technologies 2010, Oct., pp 307-315, full paper, doi:10.15221/10.307

[18] KIDSIZE project. FP7-SME-2013-606091. "Development of a new extended product-service to overcome size assignment and fitting barriers for children fashion on-line market addressing customer needs", FP7, EC. http://www.kidsizesolution.com/

[19] Rissiek, A.; Trieb, R. (2010): "iSize - Implementation of international anthropometric survey results for worldwide sizing and fit optimization in the apparel industry", Int. Conference on 3D Body Scanning Technologies, Lugano, Switzerland, 19-20 Oct. 2010, pp. 269-281. 
[20] SIZING-Sudoe Project. SOE3/P1/E482. "Red trans-regional para la transferencia tecnológica y la innovación en el sector de la moda y confección de la región SUDOE a través de la explotación de bases de datos antropométricas 3D de la población". Interreg 4B SUDOE. http://sizing-sudoe.eu/index.php/en/

[21] Shu, C.,"Making 3-D Anthropometric Data Usable", International World Engineering Anthropometry Resource Conference, 2010.

[22] Allen, B., Curless, B., \& Popović, Z. (2003): "The space of human body shapes: reconstruction and parameterization from range scans". In ACM Transactions on Graphics (TOG) (Vol. 22, No. 3, pp. 587-594). ACM.

[23] Ben Azouz, Z et al. (2005): "Extracting Main Modes of Human Shape Variation from 3-D Anthropometric Data", 5th IC3D, 2005.

[24] Shu C., et al. (2011): "Geometric and Statistical Methods for Processing 3D Anthropometric Data", Int. Symposium on Digital Human Models, 2011.

[25] Allen, B., Curless, B., \& Popovic, Z., (2004): "Exploring the space of human body shapes: Data-driven synthesis under anthropometric control". SAE Digital Human Modeling, 01-2188.

[26] Zhu, C., Byrd, R., Nocedal, J., Morales, J. L.:" L-BFGS-B".

[27] EUROFIT project - 3D homodel reconstruction from measurements. https://www.youtube.com/watch?v=adfZ3F2MX3A

[28] ISO 8559, (1989): ISO International Standard on "Garment construction and anthropometric surveys-Body dimensions".

[29] ISO 7250-1, (2008). "Basic human body measurements for technological design" - Part 1: Body measurement definitions and landmarks.

[30] http://fits.me/

[31] http://youbuy3d.org/

[32] http://corpo.myvirtualmodel.com/

[33] MakeHuman project. http://www.makehuman.org/

[34] Optitex Online Avatar. https://ws.optitex.com/optitexwebapplication/demo/snapmodel.aspx 